\title{
Case Report \\ Patients with Acromegaly Presenting with Colon Cancer: A Case Series
}

\author{
Murray B. Gordon, ${ }^{1}$ Samer Nakhle, ${ }^{2}$ and William H. Ludlam ${ }^{3}$ \\ ${ }^{1}$ Allegheny Neuroendocrinology Center, Departments of Medicine and Neurosurgery, Allegheny General Hospital, \\ 320 East North Avenue, Pittsburgh, PA 15212, USA \\ ${ }^{2}$ Palm Research Center, 9280 West Sunset Road, Suite 306, Las Vegas, NV 89148, USA \\ ${ }^{3}$ Novartis Pharmaceuticals, 1 Health Plaza, East Hanover, NJ 07936, USA \\ Correspondence should be addressed to Murray B. Gordon; mgordon740@msn.com
}

Received 27 September 2016; Accepted 13 November 2016

Academic Editor: Wayne V. Moore

Copyright (C) 2016 Murray B. Gordon et al. This is an open access article distributed under the Creative Commons Attribution License, which permits unrestricted use, distribution, and reproduction in any medium, provided the original work is properly cited.

\begin{abstract}
Introduction. Frequent colonoscopy screenings are critical for early diagnosis of colon cancer in patients with acromegaly. Case Presentations. We performed a retrospective analysis of the incidental diagnoses of colon cancer from the ACCESS trial (ClinicalTrials.gov identifier: NCT01995734). Colon cancer was identified in 2 patients (4.5\%). Case 1 patient was a 36-year-old male with acromegaly who underwent transsphenoidal surgery to remove the pituitary adenoma. After surgery, the patient underwent routine colonoscopy screening, which revealed a $40 \mathrm{~mm}$ tubular adenoma in the descending colon. A T1N1a carcinoma was surgically removed, and 1 of 22 lymph nodes was positive for metastatic disease, leading to a diagnosis of stage 3 colon cancer. Case 2 patient was a 50-year-old male with acromegaly who underwent transsphenoidal surgery to remove a $2 \mathrm{~cm}$ pituitary adenoma. The patient reported severe cramping and lower abdominal pain, and an invasive $8.1 \mathrm{~cm}^{3}$ grade 2 adenocarcinoma with signet rings was identified in the ascending colon and removed. Of the 37 lymph nodes, 34 were positive for the presence of tumor cells, and stage $3 \mathrm{c}$ colon cancer was confirmed. Conclusion. Current guidelines for colonoscopy screening at the time of diagnosis of acromegaly and at appropriate follow-up intervals should be followed.
\end{abstract}

\section{Introduction}

Acromegaly is a disease most often caused by benign somatotrophic pituitary adenomas that lead to elevated secretion of growth hormone (GH), which stimulates increased expression of insulin-like growth factor 1 (IGF-1) [1]. A persistent increase in GH and IGF-1 leads to known acromegalyassociated comorbidities, including congestive heart failure, arthritis, and impaired glucose tolerance [1]. In some studies, colon polyps or cancer have also been reported to occur more frequently in patients with acromegaly than in the general population [2]. However, data from other studies have not supported the association between acromegaly and colon cancer $[2,3]$. Here, we present case studies of 2 patients who were diagnosed with colon cancer from a small cohort of patients with acromegaly.

\section{Case Presentations}

We performed a retrospective analysis of the incidental diagnoses of colon cancer in the ACCESS trial (ClinicalTrials.gov identifier: NCT01995734), an open-label, multicenter study that allowed for expanded access to pasireotide long-acting while regulatory approval was being pursued. To be included in the cohort, patients with acromegaly must have undergone surgery to remove the pituitary tumor (unless they were not eligible for surgery or refused surgery) and had uncontrolled acromegaly, as defined by IGF-1 levels greater than the upper limit of normal and random GH greater than $1 \mathrm{ng} / \mathrm{mL}$. Patients were excluded if they had been diagnosed with active malignant disease within the last 5 years (with the possible exception of basal cell carcinoma or carcinoma in situ of the cervix). Colon cancer was diagnosed using colonoscopy, and 


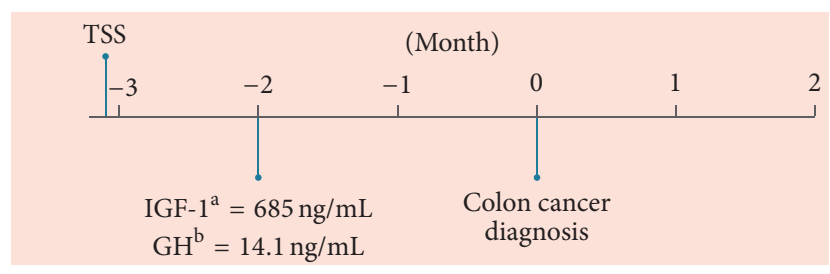

Figure 1: Patient timeline from removal of pituitary adenoma to colon cancer diagnosis. GH, growth hormone; IGF-1, insulin-like growth factor 1; TSS, transsphenoidal surgery. ${ }^{a}$ Reference range, 109-329 ng/mL. ${ }^{b}$ Reference range, $\leq 3 \mathrm{ng} / \mathrm{mL}$.

histological examination of tumor resections was used for cancer staging.

The cohort was composed of 44 patients (female, 56.8\%). The mean age was 45.5 years, and the mean body mass index was $32.9 \mathrm{~kg} / \mathrm{m}^{2}$. Impaired fasting glucose and type 2 diabetes mellitus were observed in $1(2.3 \%)$ and 9 (20.5\%) patients, respectively. Two men (4.5\%) were incidentally diagnosed with stage 3 colon cancer soon after they entered the study (after 1 week for 1 patient and after 5 months for the other patient); however, the cancer-related events were considered to be unrelated to treatment with the study drug.

Case 1 patient was a 36-year-old white male with a reported family history of colon cancer (grandmother) who presented with acromegaly, weight loss, and skin tags. The patient had not been screened by colonoscopy before diagnosis of acromegaly. Three months before case presentation, transsphenoidal surgery was performed with resection of a pituitary adenoma (Figure 1). Approximately 6 weeks after surgery, the patient's IGF-1 level was $685 \mathrm{ng} / \mathrm{mL}$, more than twice the upper limit of normal (reference range, 109$329 \mathrm{ng} / \mathrm{mL}$ ), and his $\mathrm{GH}$ level was $14.1 \mathrm{ng} / \mathrm{mL}$ (reference range, $\leq 3 \mathrm{ng} / \mathrm{mL}$ ). Magnetic resonance imaging revealed no evidence of pituitary adenoma 3 months after surgery.

The patient enrolled in the study 1 week before case presentation. One week after study enrollment, the patient underwent routine colonoscopy screening, which revealed a $40 \mathrm{~mm}$ tubular adenoma in the descending colon. A T1Nla carcinoma was surgically removed 2 weeks later; 1 of 22 lymph nodes was positive for metastatic disease. The patient was diagnosed with stage 3 colon cancer and started oxaliplatin every 3 weeks (targeting 10 treatments) and capecitabine $4 \mathrm{~g}$ once daily. The patient reported cold sensitivity, some nausea, soft stools (resolved), weight loss, metallic mouth, depression, and anxiety associated with chemotherapy treatment. The colon tumor was subsequently resected, and the patient is now in remission without evidence of tumor recurrence.

Case 2 patient was a 50 -year-old male who originally presented with headache, hypogonadism, and fatigue. Initial colonoscopy (performed 3 years before the diagnosis of acromegaly) was negative. Magnetic resonance imaging showed a $2 \mathrm{~cm}$ pituitary adenoma that was removed with transsphenoidal surgery. The patient's IGF-1 levels were uncontrolled after surgery and ranged from 244 to $583 \mathrm{ng} / \mathrm{mL}$ (reference range, 70-205 ng/mL) during 16 months of lanreotide Autogel treatment (Figure 2). The patient switched

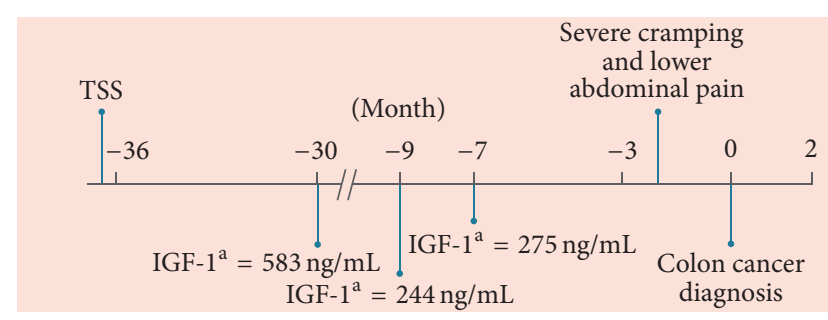

Figure 2: Patient timeline from removal of pituitary adenoma to colon cancer diagnosis. IGF-1, insulin-like growth factor 1; TSS, transsphenoidal surgery. ${ }^{a}$ Reference range, $70-205 \mathrm{ng} / \mathrm{mL}$.

to pasireotide long-acting at the beginning of the trial and continued to receive pasireotide.

Six months later, the patient reported severe cramping and lower abdominal pain, and a colonoscopy was performed. Two polyps were revealed, and an invasive $8.1 \mathrm{~cm}^{3}$ grade 2 adenocarcinoma with signet rings was removed from the ascending colon. Of the 37 lymph nodes, 34 were positive for the presence of tumor cells. Histology confirmed stage $3 c$ colon cancer, and the patient started treatment with capecitabine and oxaliplatin. The patient subsequently died in hospice care because of metastatic colon cancer. The death was not considered related to pasireotide treatment.

\section{Discussion}

These case studies detail the identification of advanced-stage colon cancer in 2 patients (4.5\%) from a cohort of patients who were being treated for uncontrolled acromegaly. The incidence of colon cancer in this cohort is comparable to and within the range of other reported epidemiological findings of colon cancer in acromegaly $(1.1 \%-20.0 \%)$ [4]. It should be noted that the identification of colon cancer was not considered to be related to the study drug, which is not surprising given that the 2 patients had only been in the study for 1 week in case 1 and 5 months in case 2, and colon cancer is associated with various stages that occur over many years. Because many patients with uncontrolled acromegaly are undiagnosed for years, it is possible that persistently elevated GH and IGF-1 levels could be a factor in the relatively high incidence of colon cancer in this cohort compared with the general population [5]. Therefore, biochemical control of GH and IGF-1 levels may reduce the risk of colon cancer in patients with acromegaly. There are currently no prospective interventional studies demonstrating to what extent therapies that reduce GH and IGF-1 levels affect the incidence and prognosis of colon cancer in acromegaly.

The extent of the association between colon cancer risk and acromegaly has been unclear. In a preclinical model, local increases in colon GH generated a tumor microenvironment that was permissive for neoplastic colon growth in mice [6]. In addition, in a meta-analysis that included 9 controlled studies of 701 patients with acromegaly and 1573 controls, there was a higher risk of colon cancer in patients with acromegaly (14/304 [4.6\%]) than in control patients $(8 / 627$ [1.2\%]) [2]. In contrast, a study by Renehan 
et al. found no difference in the prevalence of colon cancer or colonic polyps between patients with acromegaly using colonoscopy and control patients without acromegaly using autopsy examinations [7]. However, autopsy examinations may have detected a higher rate of adenomas/carcinomas in the general population because the examinations could be more extensive.

Other factors may contribute to the increased risk of colon cancer in patients with acromegaly. Hyperplastic polyps and carcinoma have been associated with higher levels of serum GH [8], although higher IGF-1 levels are also associated with increased risks $[9,10]$. In addition, the risk of colonic lesions is 2.4- to 5.8-fold higher in patients with impaired glucose regulation (impaired fasting glucose, impaired glucose tolerance, or diabetes) than in those with normal glucose tolerance [11]. This effect may be mediated by hyperinsulinemia, because the risk of developing adenomatous polyps was 14.8 times higher in patients with fasting insulin levels in the highest tertile than in those with levels in the lowest tertile [11]. Additionally, genetic factors have also been associated with more frequent incidences of colorectal cancer and may increase the risk of colorectal cancer in patients with acromegaly [12].

Acromegaly treatment guidelines from the American Association of Clinical Endocrinologists (AACE) and the Endocrine Society suggest screening for colon neoplasia with colonoscopy at the time of diagnosis of acromegaly [13, 14]. Furthermore, AACE guidelines suggest that follow-up colonoscopy be performed at time intervals "appropriate for patients at higher-than-average risk for colon cancer" [13], whereas the Endocrine Society guidelines suggest screening every 5 years for patients with elevated IGF-1 levels or polyps and every 10 years for patients with normalized IGF-1 levels and no polyps [14]. In contrast, guidelines from the British Society of Gastroenterology recommend screening every 3 years for patients with adenoma(s) at initial colonoscopy and/or elevated IGF-1, while those from the Acromegaly Consensus Group recommend screening every 3 to 5 years depending on the number and size of the adenomas [4]. These guidelines provide 3 points of consideration. First, colonoscopy was shown to be superior to fecal occult blood testing in identifying adenomas and cancer and is the suggested method of screening for colon neoplasms [15]. Second, colonoscopies should be performed at diagnosis, as evidenced in a study that showed that up to $19.3 \%$ of patients with acromegaly who were aged $<40$ years compared with $4.4 \%$ of controls had colonic neoplasia at diagnosis [10]. Third, follow-up colonoscopies should occur, although the frequency has not been firmly established. In this study, there was no evidence of colon cancer in 1 patient at his previous colonoscopy ( 3 years before the diagnosis of acromegaly and 5 years before the diagnosis of colon cancer). In a retrospective study of patients with acromegaly who were screened by colonoscopy at a mean interval of approximately every 4 years, new polyps were identified in roughly one-third of patients at each screening [16]. Additionally, in this study, a patient with a family history of colon cancer was reported. A cohort analysis previously showed a potential association between increased risk of colon cancer and acromegaly in male patients with a family history of colon cancer [17]. A joint guideline from the American Cancer Society, US Multi-Society Task Force on Colorectal Cancer, and American College of Radiology recommends that patients with a family history of colon cancer should have a colonoscopy at an earlier age (i.e., before the generally recommended age of 50 years) and more frequently than individuals at average risk [18]. Therefore, based on this case series and in conjunction with a literature review, we suggest an evidencebased guideline of follow-up screening with colonoscopy at relatively shorter intervals (e.g., every 3 years) in patients with acromegaly who are $\leq 50$ years of age, particularly in cases with a positive family history of colon cancer.

\section{Conclusion}

The diagnoses of colon cancer in this cohort of patients with acromegaly suggests that physicians should perform a routine colonoscopy screening at the time of diagnosis of acromegaly and at appropriate follow-up intervals after the diagnosis. Furthermore, control of GH and IGF-1 levels should be paramount to mitigate comorbidities such as colon cancer.

\section{Consent}

Written informed consent was obtained from one patient (case 1) for publication of this case report. A copy of the written consent is available for review on request. All reasonable efforts to obtain consent for publication from the second patient's (case 2) next of kin failed as they were untraceable. The authors have made every effort to ensure patient anonymity. There is no reason to believe that the patient would have objected to publication, and it is not felt that anyone who knew the patient would be able to identify him from the published article.

\section{Disclosure}

Dr. Ludlam is now with Chiasma, Inc, 60 Wells Avenue, Newton, MA 02459.

\section{Competing Interests}

Murray B. Gordon has served as an investigator for and received grants from Novartis Pharmaceuticals Corporation, Novo Nordisk, OPKO, and Pfizer, Inc. Samer Nakhle has received research grants from Eli Lilly, Lexicon, Novartis Pharmaceuticals Corporation, Novo Nordisk, and Sanofi. William H. Ludlam was an employee of Novartis Pharmaceuticals Corporation at the time this work was completed and is currently an employee of Chiasma, Inc.

\section{Acknowledgments}

Support for this study was provided by Novartis Pharmaceuticals Corporation. Medical editorial assistance was provided by Andrea Eckhart and Meredith MacPherson of 
MedThink SciCom (Raleigh, NC) and was sponsored by Novartis Pharmaceuticals Corporation.

\section{References}

[1] G. Lugo, L. Pena, and F. Cordido, "Clinical manifestations and diagnosis of acromegaly," International Journal of Endocrinology, vol. 2012, Article ID 540398, 10 pages, 2012.

[2] T. Rokkas, D. Pistiolas, P. Sechopoulos, G. Margantinis, and G. Koukoulis, "Risk of colorectal neoplasm in patients with acromegaly: a meta-analysis," World Journal of Gastroenterology, vol. 14, no. 22, pp. 3484-3489, 2008.

[3] P. J. Jenkins, A. Mukherjee, and S. M. Shalet, "Does growth hormone cause cancer?” Clinical Endocrinology, vol. 64, no. 2, pp. 115-121, 2006.

[4] L. Vilar, L. A. Naves, C. Caldato et al., "Acromegaly and colorectal cancer," Translational Gastrointestinal Cancer, vol. 4, no. 1, pp. 28-38, 2015.

[5] K. Lois, J. Bukowczan, P. Perros, S. Jones, M. Gunn, and R. A. James, "The role of colonoscopic screening in acromegaly revisited: review of current literature and practice guidelines," Pituitary, vol. 18, no. 4, pp. 568-574, 2015.

[6] V. Chesnokova, S. Zonis, C. Zhou et al., "Growth hormone is permissive for neoplastic colon growth," Proceedings of the National Academy of Sciences, vol. 113, no. 23, pp. E3250-E3259, 2016.

[7] A. G. Renehan, P. Bhaskar, J. E. Painter et al., "The prevalence and characteristics of colorectal neoplasia in acromegaly," The Journal of Clinical Endocrinology and Metabolism, vol. 85, no. 9, pp. 3417-3424, 2000.

[8] Y. Matano, T. Okada, A. Suzuki, T. Yoneda, Y. Takeda, and H. Mabuchi, "Risk of colorectal neoplasm in patients with acromegaly and its relationship with serum growth hormone levels," The American Journal of Gastroenterology, vol. 100, no. 5, pp. 1154-1160, 2005.

[9] P. J. Jenkins, V. Frajese, A.-M. Jones et al., "Insulin-like growth factor I and the development of colorectal neoplasia in acromegaly," The Journal of Clinical Endocrinology and Metabolism, vol. 85, no. 9, pp. 3218-3221, 2000.

[10] M. Terzolo, G. Reimondo, M. Gasperi et al., "Colonoscopic screening and follow-up in patients with acromegaly: a multicenter study in Italy," The Journal of Clinical Endocrinology and Metabolism, vol. 90, no. 1, pp. 84-90, 2005.

[11] A. Colao, R. Pivonello, R. S. Auriemma et al., "The association of fasting insulin concentrations and colonic neoplasms in acromegaly: a colonoscopy-based study in 210 patients," The Journal of Clinical Endocrinology \& Metabolism, vol. 92, no. 10, pp. 3854-3860, 2007.

[12] M. L. Torre, G. T. Russo, M. Ragonese et al., "MTHFR C677T polymorphism, folate status and colon cancer risk in acromegalic patients," Pituitary, vol. 17, no. 3, pp. 257-266, 2014.

[13] L. Katznelson, J. L. D. Atkinson, D. M. Cook et al., "American Association of Clinical Endocrinologists medical guidelines for clinical practice for the diagnosis and treatment of acromegaly-2011 update: executive summary," Endocrine Practice, vol. 17, no. 4, pp. 636-646, 2011.

[14] L. Katznelson, E. R. Laws Jr., S. Melmed et al., "Acromegaly: an endocrine society clinical practice guideline," The Journal of Clinical Endocrinology and Metabolism, vol. 99, no. 11, pp. 39333951, 2014.
[15] F. Bogazzi, M. Lombardi, I. Scattina et al., "Comparison of colonoscopy and fecal occult blood testing as a first-line screening of colonic lesions in patients with newly diagnosed acromegaly," Journal of Endocrinological Investigation, vol. 33, no. 8, pp. 530-533, 2010.

[16] D. Dworakowska, M. Gueorguiev, P. Kelly et al., "Repeated colonoscopic screening of patients with acromegaly: 15-year experience identifies those at risk of new colonic neoplasia and allows for effective screening guidelines," European Journal of Endocrinology, vol. 163, no. 1, pp. 21-28, 2010.

[17] J. E. Brunner, C. C. Johnson, S. Zafar, E. L. Peterson, J. F. Brunner, and R. C. Mellinger, "Colon cancer and polyps in acromegaly: increased risk associated with family history of colon cancer," Clinical Endocrinology, vol. 32, no. 1, pp. 65-71, 1990.

[18] E. C. Cabebe, Colorectal Cancer Guidelines, Medscape, http:// emedicine.medscape.com/article/2500006-overview. 


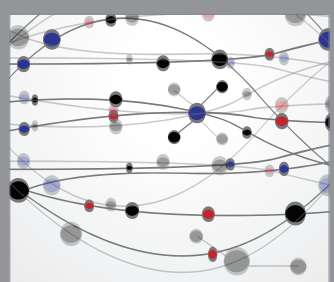

The Scientific World Journal
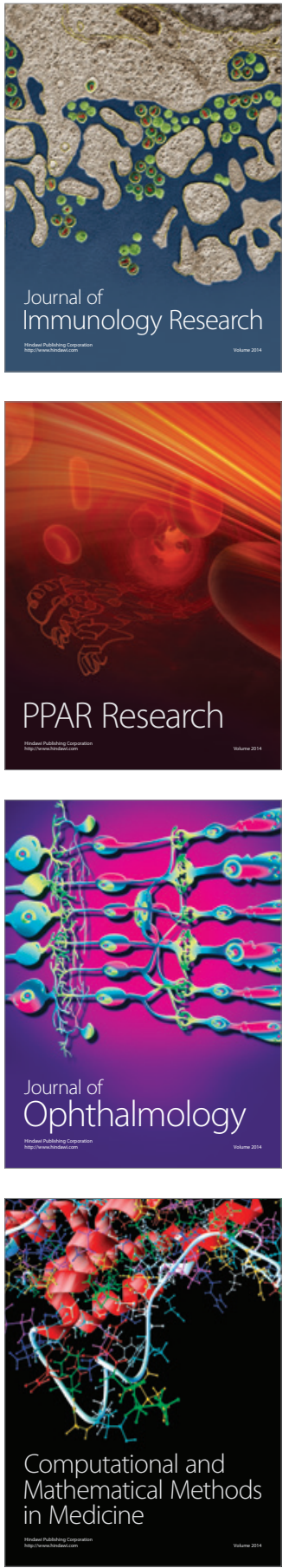

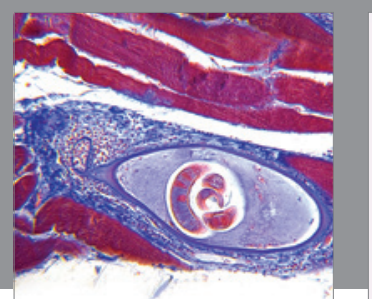

Gastroenterology Research and Practice

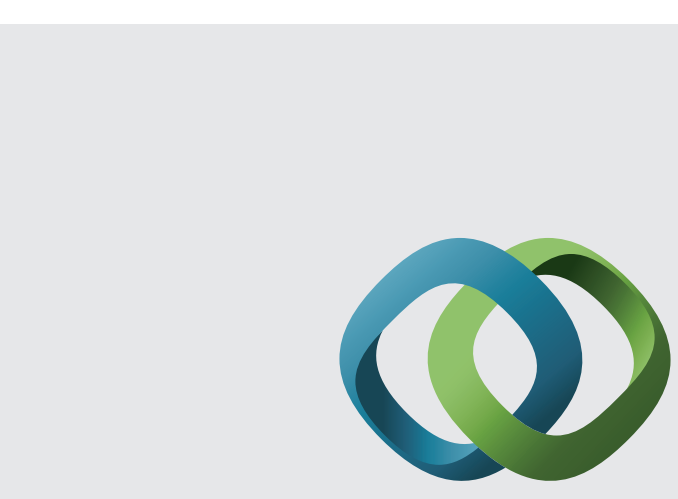

\section{Hindawi}

Submit your manuscripts at

http://www.hindawi.com
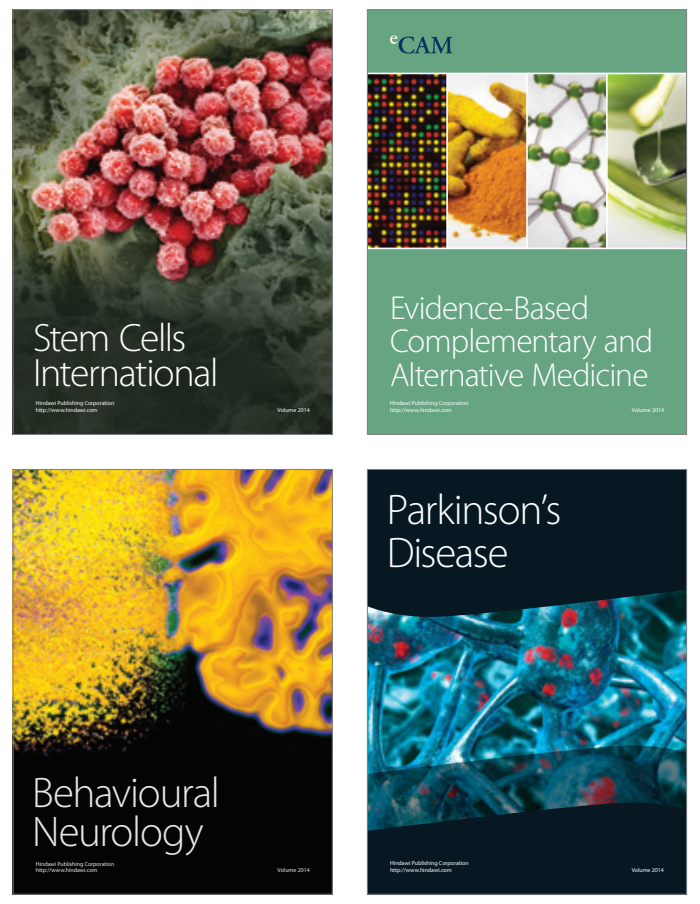
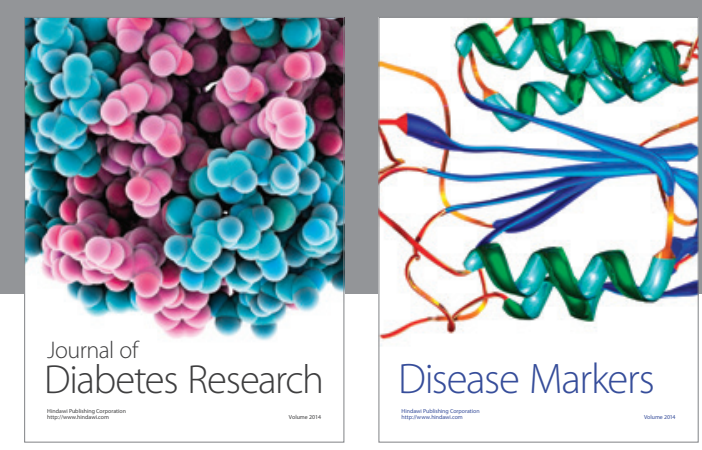

Disease Markers
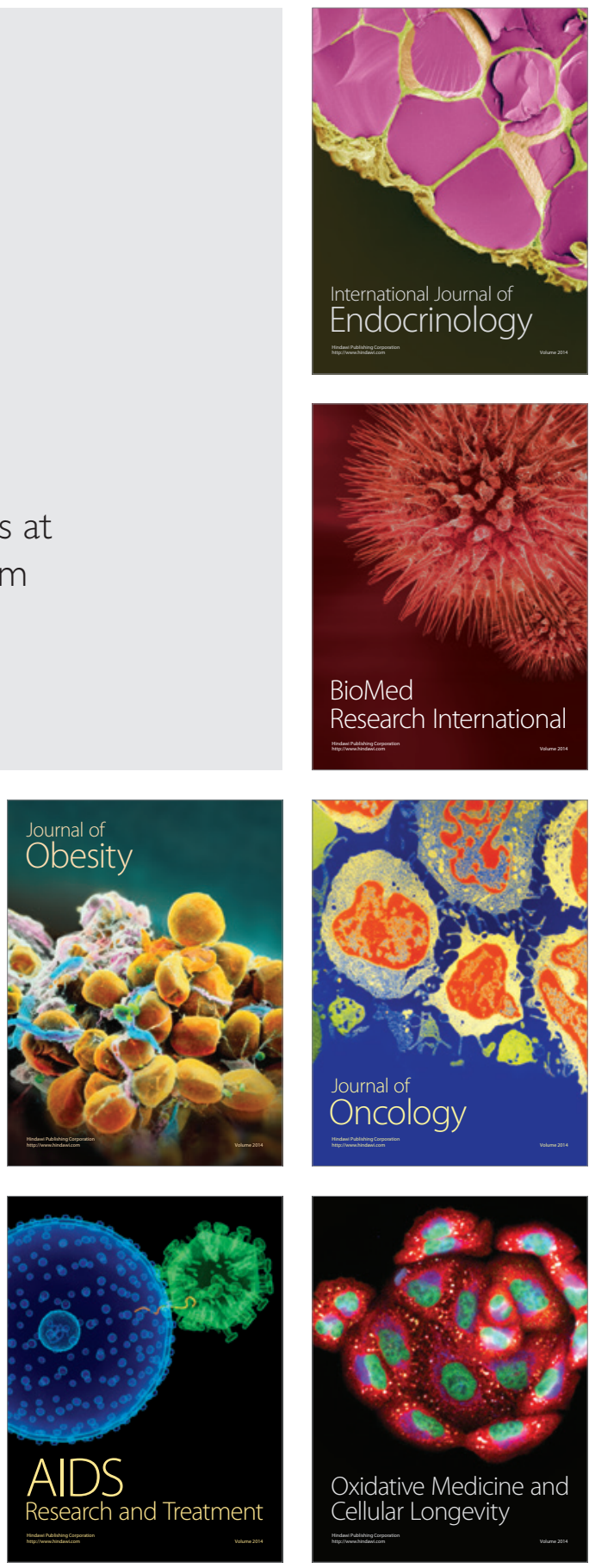